\title{
STABILITY OF A COLLISIONAL GALACTIC DISK
}

\author{
E. GRIV
}

Department of Physics, Ben-Gurion University, Israel

Until recently, only collisionless models have been investigated in the kinetic treatment of stellar disk stability (e.g., Fridman and Polyachenko [1984]). This is due to the fact that the frequency of ordinary binary stellar gravitational (elastic) encounters in the Galaxy is much smaller than the variation of the gravitational field for the process being studied. However, in the pioneering paper Spitzer and Schwarzschild (1951) proposed a different kind of encounter: interaction of stars with gas clouds of the interstellar medium having a mass of rougly $10^{6} M_{\odot}$. In recent years this hypothesis was partially confirmed by observations: it was discovered in the Galaxy a few thousand giant molecular clouds of mass $M_{c} \geq 10^{5} M_{\odot}$. Other evidence of dynamical relaxation of the star-cloud disk in the solar neighborhood was found by Grivnev and Fridman (1990); the time of relaxation was estimated equal to $\tau=(2-4) \times 10^{9}$ years. Hence the study of collisional star-cloud system is not only of academic interest - on the time span $t \geq 10^{9}$ years an actual galaxy may be a collisional ensemble of stars and clouds.

Here I report the results of the theoretical study of the dynamics of a stellar galactic disk when star-cloud collisions are taken into account (Griv and Peter 1994, Astrophys. J., submitted). With respect to the physical state of our own Galaxy, the dynamics of a system with rare encounters between stars and clouds is considered when the epicyclic frequency $\kappa \simeq$ $10^{-8}$ encounters $\nu_{c} \simeq 1 / \tau \sim 10^{-9}$ years $^{-1}$. In addition, the proper, but complicated, collision integral was replaced by an approximate model of Bhatnagar et al. (1954).

The dispersion relation, which connects the oscillation frequency $\omega$ and the wavenumber $k$, was obtained in the form

$\frac{\kappa^{2} x^{2}}{2 \pi G \sigma_{0}|k|}=1-\sum_{l=-\infty}^{\infty} \frac{\omega_{*} e^{-x} I_{l}(x)}{\omega_{*}-l \kappa+i \nu_{c}}+2 \Omega \frac{m x^{2}}{r L k^{2}} \frac{e^{-x} I_{0}(x)}{\omega_{*}+i \nu_{c}}-\frac{i \nu_{c} \kappa^{2} x^{2}}{2 \pi G \sigma_{0}|k|} \frac{e^{-x} I_{0}(x)}{\omega_{*}+i \nu_{c}}$ 
where $x=k^{2} c_{r}^{2} / \kappa^{2}, c_{r}$ is the radial-velocity dispersion, $\sigma_{0}$ is the equilibrium surface density of the stellar disk, $L$ is the scale of density inhomogeneity, $\omega_{*}=\omega-m \Omega, m$ is the azimuthal mode number (the number of spiral arms), and $I_{l}(x)$ is the modified Bessel function.

The dispersion relation describes the Jeans and gradient branches of oscillations modified by collisions. Since in general, this relation is very complicated, in order to deal with the most interesting oscillation types I consider only some limiting cases of perturbations. Thus from the above relation, in the case of weak collisions, $\omega_{*}^{2} \gg \nu_{c}^{2}$, the dispersion law for the Jeans branch is described by

$$
\omega_{* 1,2} \simeq \pm p\left|\omega_{J}\right|-\frac{\kappa^{2}}{2 \omega_{J}^{2} x^{2}}\left(i \nu_{c}+2 \Omega \frac{m x^{2}}{r L k^{2}}\right),
$$

where $p=1$ for perturbations with $\omega_{J}^{2}>0$ and $p=i$ for perturbations with $\omega_{J}^{2}<0$, and $\omega_{J}$ is the frequency of the Jeans oscillations. From (1) one concludes that Jeans-stable perturbations $\left(\omega_{J}^{2}>0\right)$ will decay $\left(\operatorname{Im}\left\{\omega_{*}\right\}>0\right)$ in the presence of collisions, and Jeans-unstable perturbations $\left(\omega_{J}^{2}<0\right)$ will undegro additional weak destabilization $\left(\operatorname{Im}\left\{\omega_{*}\right\}<0\right)$.

Apart from the Jeans roots (1), the dispersion relation has another root, which describes the gradient branch of oscillations

$$
\omega_{*} \simeq\left(2 \Omega \frac{m x^{2}}{r L k^{2}}+i \nu_{c}\right) \frac{\kappa^{2}}{\omega_{J}^{2}} \frac{I_{0}(x)}{I_{1}(x)} .
$$

Accordingly, the gradient perturbations are unstable in the presence of particle encounters in the Jeans-stable disk. In principle, this dissipative instability, as well as the kinetic one, can be considered as generating mechanisms for unstable short-scale spiral density waves.

I would like to acknowledge the support from the Institute of Astronomy of National Central University, Chung-Li and the NSC in Taiwan, ROC.

\section{References}

Bhatnagar, P.L., Gross, E.P. and Krook, M. (1954) Phys. Rev., 94, p. 511

Fridman, A.M. and Polyachenko, V.L. (1984) Physics of Gravitating Systems, Vols. 1 and 2, Springer-Verlag, New York

Grivnev, E.M. and Fridman, A.M. (1990) Soviet Astron., 34, p. 10

Spitzer, L. and Schwarzschild, M. (1951) Astrophys. J., 114, p. 385. 